\title{
A Novel Fault-Tolerant Control Strategy for Near Space Hypersonic Vehicles via Least Squares Support Vector Machine and Backstepping Method
}

\author{
Jia Song and Jiaming Lin \\ School of Astronautics \\ Beihang University \\ Beijing, China \\ songjia@buaa.edu.cn
}

\author{
Erfu Yang \\ Space Mechatronic Systems Technology Laboratory \\ Strathclyde Space Institute, University of Strathclyde \\ Glasgow, UK \\ erfu.yang@strath.ac.uk
}

\begin{abstract}
Near Space Hypersonic Vehicle (NSHV) could play significant roles in both military and civilian applications. It may cause huge losses of both personnel and property when a fatal fault occurs. It is therefore paramount to conduct fault-tolerant research for NSHV and avoid some catastrophic events. Toward this end, this paper presents a novel fault-tolerant control strategy by using the LSSVM (Least Squares Support Vector Machine)-based inverse system and Backstepping method. The control system takes advantage of the superiority of the LSSVM in solving the problems with small samples, high dimensions and local minima. The inverse system is built with an improved LSSVM. The adaptive controller is designed via the Backstepping which has the unique capability in dealing with nonlinear control systems. Finally, the experiment results demonstrate that the proposed method performs well.
\end{abstract}

Keywords-Near space hypersonic vehicle; least squares support vector machine; inverse system control; fault-tolerant control

\section{INTRODUCTION}

In recent years, it has been widely concerned that the NSHV has achieved the purpose of long-range precision for its potential applications in both military and civilian areas[1]. One challenge in its applications is that many faults may occur, which could cause the casualties and property loss. To avoid these kind of catastrophic consequences, it is significant to carry out research on the fault-tolerant control technology for NSHV. With the development of aerospace technology and the improving complexity of aerospace systems, many nonlinear control methods and intelligent approaches, such as Gain Scheduling (GS), Adaptive Control (AC), and Backstepping method, etc, have been introduced. [2].

In the GS method, some typical working point are first chosen within the flight envelope. Then they are balanced and linearized at each working point. The sub-controllers are accordingly designed for every linearized subsystem. Finally the controller structure and corresponding gain in all kinds of fault cases are achieved offline. In the process of flight, the fault is first classified and judged by the Fault Detection and Diagnosis (FDD) system .Then the vehicle is dealt with different faults through the controller designed in advance. So the GS method can only

Funded by the National Science Foundation of China (Grant No. 51206007), the Aeronautical Science Foundation of China (Grant No. 2013ZC51) and the China Scholarship Council (CSC) (Grant No.20140625104) invoke the fault-tolerant controllers designed in advance. No more need is required for parameter estimation and system identification. This method responses very fast, but in [11] it indicated that, for the vehicle with high coupling and high mobility, the parameters can change suddenly when switching over different working point. Since it is difficult to build a satisfactory dispatching function, the stability of the NSHV cannot be guaranteed when using the GS method.

The AC method is based on the theory of Model Reference Adaptive Control. In the failure situation, the adaptive controller can adjust the relevant parameters to rebuild the controller by tracking the output of reference model. Without fault detection and diagnose, the reconstruction system can achieve the purpose of fault-tolerant control quickly and stably. Hence, it is widely used in nonlinear system control [12-15]. But the AC method is a nonlinear controller in essence, so it is difficult to implement in real physical system.

The Backstepping is a recursive design method [16-17,19]. It needs to simplify the nonlinear system in strict feedback form of differential equations early in the controller design. After specifying the control objectives, the virtual control variables are introduced recursively for each subsystems by using the Lyapunov stability theory until the input signals can drive control system directly. The outputs can track the inputs of the control system, and achieve the purpose of vehicle control. Because of the characteristics of Backstepping design, it is no longer required that uncertain system satisfies the matched condition. Along with Lyapunov function, the Backstepping method makes it very convenient to analyze the stability of nonlinear systems and design the controller. But because it is necessary to design a virtual controller for every subsystem, the Backstepping method will need a long time to adjust the parameters of every subsystem when dealing with higher-order system. It implies a large amount of calculations, which sometimes occurs in differential blast. The Backstepping method has been applied in fault-tolerant control along with other methods such as adaptive control, neutral network, LSSVM and so on [16][17]. Among them, the LSSVM has some unique advantages to solve the pattern recognition problems with small samples and nonlinear and high dimensions [3] [4]. Moreover, the LSSVM is simple and can have high stable precision [18]. 
Taking the advantages and disadvantages of these existing fault-tolerant control methods into account, this paper presents a new fault-tolerant control scheme which is based on an inverse model using an improved LSSVM and Backstepping in case of that the actuator failures are known. The proposed approach identifies $\alpha$ step inverse system combined with the LSSVM, and the inverse system which is in the series with the controlled system. It achieves the identity mapping between the desired output and the actual output of the system. Compared with the classical control methods, this new scheme performs significantly faster with the better controller stability.

\section{Flight MOdel of NeAR SPACE HyPERSONIC VeHICle}

This paper uses the near space hypersonic vehicle (NSHV) as the control plant [5]. The aerodynamic equations and model parameters are obtained from [6]. The atmospheric model is referred to the U.S. standard atmosphere 1976. The three-view drawing is shown in Fig.1

The developed model of the NSHV has considered the complex NSHV flight environment, changeable pneumatic parameter, high Mach numbers, etc. It uses the following twelve nonlinear differential equations to describe the unpowered reentry of the NSHV:

$$
\begin{gathered}
\dot{x}=V \cos \gamma \cos \chi \\
\dot{y}=V \sin \gamma \\
\dot{z}=-V \cos \gamma \sin \chi \\
\dot{V}=-\frac{D}{m}+\frac{g}{r_{h}}[x \cos x \cos y+(y+R) \sin \gamma-z \sin x \operatorname{con} y] \\
\dot{\gamma}=\frac{L \cos \mu-Y \sin \mu}{m V \cos \chi}+\frac{g}{r_{h} V \cos \chi} \\
\dot{\chi}=-\frac{Y \cos \chi \sin \gamma+(y+R) \cos \gamma+z \sin \chi \sin \gamma]}{m V \cos \mu}-\frac{g}{r_{h} V}(x \sin \chi+z \cos \chi) \\
\dot{\alpha}=q-p \cos \alpha \tan \beta+r \sin \alpha \tan \beta \\
-\frac{1}{m V \cos \beta}(L+m g \cos \gamma \cos \mu) \\
\dot{\beta}=p \frac{\cos \alpha}{\cos \beta}-r \frac{\sin \alpha}{\cos \beta}+\frac{1}{m V}\lceil L(\sin \gamma \sin \mu+\tan \beta) \\
+Y \sin \gamma \cos \mu+m g \cos \gamma \cos \mu \tan \beta]
\end{gathered}
$$
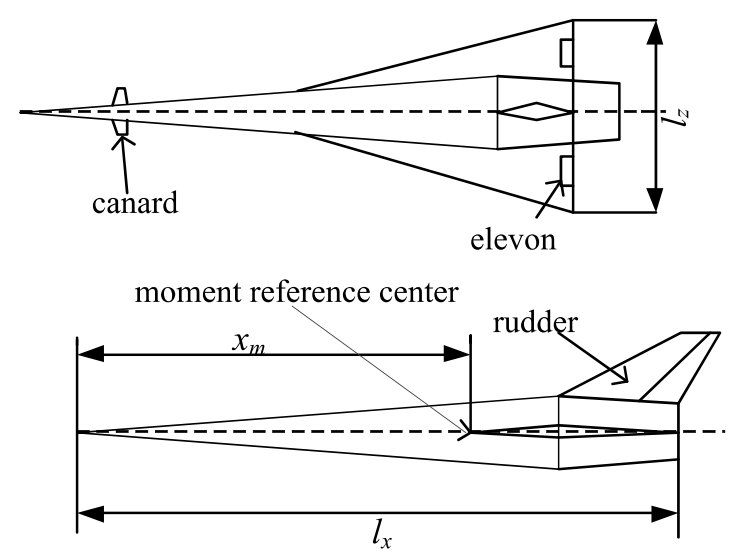

Fig. 1. Three view of the NSHV.

$$
\begin{aligned}
& \dot{p}=\frac{I_{y}-I_{z}}{I_{x}} r q+\frac{1}{I_{x}} \bar{l} \\
& \dot{r}=\frac{I_{z}-I_{x}}{I_{y}} p q+\frac{1}{I_{y}} \bar{m} \\
& \dot{q}=\frac{I_{x}-I_{y}}{I_{z}} r p+\frac{1}{I_{z}} \bar{n}
\end{aligned}
$$

In this paper we mainly discuss about pyramidal NSHV which controls the attitude through elevators, rudder and ailerons during reentry. It is necessary to build mathematical model for the three kinds of rudder based on high credibility. The accuracy of modeling the force and torque are also very important. The aerodynamic force and moment coefficients in the fitting polynomials are given by:

$$
\left\{\begin{array}{l}
D=C_{D} \rho V^{2} S / 2=C_{D} q S \\
L=C_{L} \rho V^{2} S / 2=C_{L} q S \\
Y=C_{Y} \rho V^{2} S / 2=C_{Y} q S
\end{array}\right.
$$

Where, $\rho=1.225 e^{-\frac{h}{7200.1}}$ is the atmospheric density, $S$ is the reference area of wing surface, $v$ is the flight speed, $q$ is the kinetic pressure.

$$
\left\{\begin{array}{l}
C_{D}=C_{D, \alpha}+C_{D, \delta_{e}} \delta_{e}+C_{D, \delta_{a}} \delta_{a}+C_{D, \delta_{r}} \delta_{r} \\
C_{L}=C_{L, \alpha}+C_{L, \delta_{e}} \delta_{e}+C_{L, \delta_{a}} \delta_{a} \\
C_{Y}=C_{Y, \beta} \beta+C_{Y, \delta_{e}} \delta_{e}+C_{Y, \delta_{a}} \delta_{a}+C_{Y, \delta_{r}} \delta_{r}
\end{array}\right.
$$

Where, $\delta_{e} 、 \delta_{a} 、 \delta_{r}$ are the drift angles of left and right elevator, and rudder, respectively. $\bar{l} 、 \bar{m} 、 \bar{n}$ are rolling moment, yawing moment and pitching moment, and defined as: 


$$
\left\{\begin{array}{l}
\bar{l}=C_{l} b S \rho V^{2} / 2 \\
\bar{m}=C_{m} b S \rho V^{2} / 2+X_{c g} Y \\
\bar{n}=C_{n} c S \rho V^{2} / 2+X_{c g}(D \sin \alpha+L \cos \alpha)
\end{array}\right.
$$

Where, $b$ is the wing span, $c$ is the average aerodynamic chord, $C_{l} 、 C_{m}, C_{n}$ are the torque coefficients of roll, yaw, and pitch as given below $\bar{l} 、 \bar{m}, \bar{n} \mathrm{t}$

$$
\left\{\begin{array}{l}
C_{\bar{l}}=C_{\bar{l}, \beta} \beta+C_{\bar{l}, \delta_{e}} \delta_{e}+C_{\bar{l}, \delta_{a}} \delta_{a}+C_{\bar{l}, \delta_{r}} \delta_{r}+C_{\bar{l}, p} \frac{p b}{2 V}+C_{\bar{l}, r} \frac{r b}{2 V} \\
C_{\bar{m}}=C_{\bar{m}, \beta} \beta+C_{\bar{m} \delta_{e}} \delta_{e}+C_{\bar{m} \delta_{a}} \delta_{a}+C_{\bar{m} \delta_{r}} \delta_{r}+C_{\bar{m}, p} \frac{p b}{2 V}+C_{\bar{m}, r} \frac{r b}{2 V} \\
C_{\bar{n}}=C_{\bar{n}, \alpha}+C_{\bar{n}, \delta_{e}} \delta_{e}+C_{\bar{n}, \delta_{a}} \delta_{a}+C_{\bar{n}, \delta_{r}} \delta_{r}+C_{\bar{n}, q} \frac{q c}{2 V}
\end{array}\right.
$$

All the aerodynamic coefficients are the functions of the angles of attack and the Mach numbers, which change sharply along with the change of the flight speed and attitude. This phenomena makes the controller design of NSHV more complex and difficult. In addition, the nonlinear function in NSHV model is very complicated and the coupling degree of is also high, which significantly increases the difficulty in controller design.

\section{FAULT-TOLERANT CONTROLLER DESIGN FOR REENTRY ATTITUDE}

\section{A. Reentry attitude controller design of Near Space Hypersonic Vehicle based on Least Squares Support Vector Machine}

In this section, an adaptive fault-tolerant controller for the reentry attitude control of NSHV is designed with the method of error compensation through the LSSVM and Backstepping method. In the Backstepping method the virtual controller is in essence a kind of error compensator, from inside to outside by tracking the system instructions step by step to ensure the equanimity of control plant. So this method suits for the attitude controller design of the NSHV as the system has strict feedback of parameters.

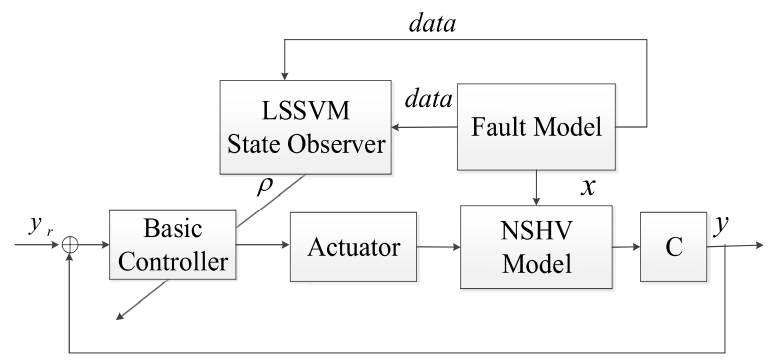

Fig. 2. The flow chart of the fault-tolerance controller.

By joining the fault information with the strict feedback of reentry attitude, the nonlinear mathematical models are built. The
Backstepping adaptive controller is used as the basic controller in the NSHV control system to track the input and output. When a failure in the flight system occurs, the state observer approaches the fault message by online learning. The observer identifies the system, and then adds the fault identification result into the basic controller. And then the stability of the closed-loop system is judged by using a Lyapunov function. Till now, the design of the fault-tolerant control of the NSHV attitude is completed. Fig. 2 depicts the flowchart of the proposed fault-tolerant controller.

When there is no fault or external interference in the system, $\rho=0$, the basic controller can ensure the stability of the NSHV control system. While a fault occurs, the system performance declines, the system fault and uncertain noises are brought into the LSSVM. Then the identification result is added into the basic controller in the form of compensation to realize the fault-tolerant control of the NSHV. Because it takes some time to identify the fault through the LSSVM, before the compensation information is added, the basic controller needs to guarantee the stability of control system to the greatest extent.

\section{B. Reentry attitude observer design of Near Space Hypersonic \\ Vehicle based on Least Squares Support Vector Machine}

In order to facilitate the observer design, the nonlinear state equations for the reentry attitude control of the NSHV are combined with fault information as follows:

$$
\dot{x}(t)=f\left(x_{1}, t\right)+g(x, t) v(t)+d(x, v, t)+u(x, v, t)
$$

Where, $x(t) \in R^{n}$ is the attitude angle and the state of angle velocity, $v(t) \in R^{m}$ is the input of control torques, $u(x, v, t)$ is the system fault, $d(x, v, t) \in R^{n}$ is the $\mathrm{u}$ external uncertainty.

If $d(x, v, t)$ is bounded, and $\left|d_{i}\right| \leq \bar{d}_{i}(i=1, \ldots, n)$, the system is observable.

For the nonlinear control system of the NSHV reentry attitude described above, this paper proposes a nonlinear observer based on the strong ability of function approximation of the LSSVM and regression theory. It is described as follows:

$$
\hat{\hat{x}}(t)=f\left(x_{1}, t\right)+g(x, t) v+G\left(x(t)-\hat{x}_{1}(t)\right)+\hat{u}(x, v, t ; \alpha, b)
$$

Where, $u(x, v, t ; \alpha, b)$ is the estimation of the fault occurring to the NSHV system by the LSSVM regression via the online training. According to (18), $\alpha, b$ are decided by the training result, it is used as a Langrage multiplier vector and $G$, the constant deviation in the LSSVM regression, the Hurwitz matrix.

The state estimation error is defined as $\varepsilon(t)=x-\dot{x}$. It yields

$$
\dot{e}(t)=G e(t)+(u(x, v, t)-\hat{u}(x, v, t ; \hat{\alpha}, \hat{b}))
$$

And the observer error between the estimation and reality is: 
$\|u(x, v, t)-\hat{u}(x, v, t ; \hat{\alpha}, \hat{b})\| \leq \xi$. Where, $\xi$ is the upper bound of the estimation error.

According to the nonlinear equations of the control system and the mathematic form of the observer, if $G$ is Hurwitz matrix, then $Q$ is the positive definite symmetric matrix, and there must be a positive definite symmetric matrix $P$, which meets with Lyapunov equation:

$$
G P+P G^{T}=-Q
$$

Hence the estimation error from the observer must be uniformly bounded. Its proof is provided as follows:

Firstly the Lyapunov function is chosen as $V(t)=e^{T} \tilde{P} e$. The equations are derived, to obtain the following results:

$$
\begin{aligned}
& V(t)=\dot{\varepsilon^{T}} \tilde{P} e+e^{T} \tilde{P} e \\
&=\left[G e+(d(x, v, t)+(u(x, v, t)-\hat{u}(x, v, t ; \dot{\alpha}, \dot{b}))]^{T} \tilde{P} e\right. \\
&+e^{T} P[G e+(u(x, v, t)-\hat{u}(x, v, t ; \dot{\alpha}, \dot{b})) \tilde{P} e] \\
&=e^{T}\left(G^{T} P+P G\right) e+2[u(x, v, t)-\hat{u}(x, v, t ; \dot{\alpha}, \dot{b})]^{T} \tilde{P} e \\
& \leq-e^{T} \tilde{Q} e+2\left(\eta_{0}+\xi\right) \tilde{P} e \\
& \leq-\|e\|^{2} \lambda_{\min }(\mathrm{Q})+2\left(\eta_{0}+\xi\right) \lambda_{\max }(P)\|e\|
\end{aligned}
$$

It is obvious that, when $\|e\|>\frac{2\left(\eta_{0}+\xi\right) \lambda_{\text {max }}(P)}{\lambda_{\text {min }}(\mathrm{Q})}, V(t)<0$. The proof is completed.

The LSSVM uses the estimation technique by online training. The input is the quantity of state $x$, the output is the estimated value of the system fault. Any unknown fault of the system can be estimated in theory. Because we are blind to system faults, the initial parameter $\hat{\alpha}, \hat{b}$ are set to meet the condition $u(x, v, t ; \hat{\alpha}, \hat{b})$ $=0$. The sample update condition is: $\|e\|>\tilde{\varepsilon}$

Where, $\tilde{\varepsilon}$ is the threshold value.

$$
\tilde{\varepsilon}=\int_{0}^{\infty}\|\exp (G t)\|\left\|\eta_{0}\right\| d t
$$

The latest samples are used in this paper to update the oldest sample in the training process with the LSSVM.

\section{Feedback model of Near Space Hypersonic Vehicle Reentry Attitude}

The model of reentry attitude of the NSHV is built as:

$$
\left\{\begin{array}{l}
\dot{x}_{1}-f_{1}\left(x_{1}\right)+g_{11}\left(x_{1}\right) x_{2}+g_{12}\left(x_{1}\right) \delta \\
\dot{x}_{2}=f_{2}\left(x_{1}, x_{2}\right)+g_{2}\left(x_{1}\right) \delta
\end{array}\right.
$$

Where, $x_{1}$ is the attitude angle vector, $x_{2}$ is the angle velocity vector, $g_{11}(x)$ is the coupling relation between $x_{1}$ and $x_{2}, g_{12}\left(x_{1}\right)$ is the distributed moment of the control torque to the deflection of the rudder surface, which can be ignored in practice. $g_{2}\left(x_{1}\right)$ is the coupling relation between $x_{2}$ and the rudder deflection. For the convenience of the fault-tolerant controller design through the Backstepping method, the attitude model can be transformed into the following strict feedback equation:

$$
\left\{\begin{array}{l}
\dot{x}_{1}=f_{1}\left(x_{1}\right)+g_{11}\left(x_{1}\right) x_{2} \\
\dot{x}_{2}=f_{2}\left(x_{1}, x_{2}\right)+g_{2}\left(x_{1}\right) u
\end{array}\right.
$$

Where, $u=\delta$ is the rudder deflection vector.

Equation (23) indicates that, when the rudder angle fault occurs, the attitude angle velocity will be the first to be influenced. Therefore when the actuators suddenly break down, $x_{2}$ will be influenced first, then $x_{1}$ is influenced by $x_{2}$.Taking it into account, at the time of $t_{n}$, the parameter of the NSHV is uncertain or influenced by the external disturbance, (23) can be transformed into the following form with the combined interference for adding the compensation information into the base controller:

$$
\left\{\begin{array}{l}
\dot{x}_{1}=f_{1}\left(x_{1}\right)+g_{11}\left(x_{1}\right) x_{2}+\rho_{1}\left(x_{1}, x_{2}, t\right) \\
\dot{x}_{2}-f_{2}\left(x_{1}, x_{2}\right)+g_{2}\left(x_{1}\right)\left(u+u_{n}\right)-f_{2}\left(x_{1}, x_{2}\right)+ \\
g_{2}\left(x_{1}\right) u+\rho_{2}\left(x_{1}, x_{2}, t\right)
\end{array}\right.
$$

Where, $\rho_{1}\left(x_{1}, x_{2}, t\right)$ is the external interference happening to subsystem $x_{1}, \rho_{2}(x)=g_{2}\left(x_{1}\right) u_{n}$ is the influence of the fault information imposed to the subsystem $x_{2}, u_{n}$ is the fault information occurring in the NSHV at the time $t_{n}$.

\section{The adaptive controller design for Near Space Hypersonic \\ Vehicle with Backstepping Methods}

The adaptive controller is designed through the use of the Backstepping methods. It aims to ensure the system performs stalely when the system develops into a fault mode. The adaptive controller increases the cushion time for compensating the faulttolerant controller to track the expected attitude angle signal $x_{1 d}=\left[\alpha_{d}, \beta_{d}, \mu_{d}\right]^{T}$.

To facilitate the design and analysis, two assumptions are made in the following.

Assumption 1: The signal of the expected attitude angle 
$x_{1 d}=\left[\alpha_{d}, \beta_{d}, \mu_{d}\right]^{T}$ is bounded, i.e.:

$$
\left\|\left[x_{1 d}, \dot{x}_{1 d}, \ddot{x}_{1 d}\right]\right\| \leq C_{d}
$$

Where, $C_{d} \in R$ is a positive constant, $\|\bullet\|$ means the 2-norm of a matrix or vector.

Assumption 2: There exist positive constants $\alpha_{m}, \beta_{m}, \mu_{m} \in R$, $\alpha, \beta, \mu$ meet $|\alpha| \leq \alpha_{m},|\beta| \leq \beta_{m}$, and $|\mu| \leq \mu_{m}$, while $g_{11}\left(x_{1}\right), g_{2}\left(x_{1}\right)$ are reversible for them, and there exists $\left|g^{-1}{ }_{11}\left(x_{1}\right)\right| \leq C_{g_{11}^{-1}}, \rho_{1}$ is bounded, and $\left\|\rho_{1}\right\| \leq c_{\rho_{1}}$.

Step 1: Define a tracking error of the subsystem $x_{1}$ as $e_{1}=x_{1}-x_{1, d}$, where $x_{1, d}$ is the expected state of the system. Taking the derivative of $e_{1}$ gives:

$$
\dot{e}_{1}=\dot{x}_{1}-\dot{x}_{1, d}=f_{1}\left(x_{1}\right)+g_{11}\left(x_{1}\right) x_{2}+\rho_{1}(x)-\dot{x}_{1, d}
$$

Now the stability of the closed-loop system is judged with a Lyapunov function. Let us define the Lyapunov function as: $V_{1}=\frac{1}{2} e_{1}^{T} e_{1}$, then take its derivative, we have:

$$
\left.\dot{V}_{1}=e_{1}^{T} \dot{e}_{1}=e_{1}^{T}\left\lceil f_{1}\left(x_{1}\right)+g_{11}\left(x_{1}\right) x_{2}+\rho_{1}(x)-\dot{x}_{1, d}\right)\right\rceil
$$

Define the virtual control vector as:

$$
x_{2, d}=g_{11}^{-1}\left(x_{1}\right)\left\lceil-k_{1} e_{1}-f_{1}\left(x_{1}\right)-\rho_{1}(x)+\dot{x}_{1, d}\right\rceil
$$

Where, $x_{2}$ is the expected angle velocity, $k_{1}$ is the diagonal gain matrix, $k_{1}=\operatorname{diag}\left\{k_{11}, k_{12}, k_{13}\right\}$, and $k_{11}, k_{12}, k_{13}>0$, then the error of the subsystem $x_{2}$ is defined as:

$$
e_{2}=x_{2}-x_{2, d}
$$

By inserting (28) and (29) into (27), we have:

$$
\begin{aligned}
\dot{V}_{1} & -e_{1}^{T}\left[f_{1}\left(x_{1}\right)+g_{11}\left(x_{1}\right)\left(e_{2}+x_{2, d}\right)+\rho_{1}(x)-\dot{x}_{1, d}\right] \\
& =e_{1}^{T}\left[f_{1}\left(x_{1}\right)+g_{11}\left(x_{1}\right) e_{2}+\left(-k_{1} e_{1}-f_{1}\left(x_{1}\right)-\rho_{1}(x)\right.\right. \\
& \left.\left.+\dot{x}_{1, d}\right)+\rho_{1}(x)-\dot{x}_{1, d}\right] \\
& =e_{1}^{T}\left[-k_{1} e_{1}+g_{11}\left(x_{1}\right) e_{2}\right]=-k_{1}\left\|e_{1}\right\|^{2}+e_{1}^{T} g_{11}\left(x_{1}\right) e_{2}
\end{aligned}
$$

When $e_{2}=0, x_{2, d}=x_{2}, \dot{V}_{1} \leq 0$, it is necessary to keep the working of the designed virtual controller.

Step 2: Take the derivative of (29):

$$
\begin{aligned}
\dot{e}_{2} & =\dot{x}_{2}-\dot{x}_{2, d} \\
& =f_{2}\left(x_{1}, x_{2}\right)+g_{2}\left(x_{1}\right)\left(u+u_{n}\right) \\
& =f_{2}\left(x_{1}, x_{2}\right)+g_{2}\left(x_{1}\right) u+\rho_{2}(x)-\dot{x}_{2, d}
\end{aligned}
$$

The second Lyapunov function is chosen as:

$$
V_{2}=V_{1}+\frac{1}{2} e_{2}^{T} e_{2}=\frac{1}{2} e_{1}^{T} e_{1}+\frac{1}{2} e_{2}^{T} e_{2}
$$

Let us take the derivative of (32), we have:

$$
\begin{aligned}
& \dot{V}_{2}-\frac{\partial V_{2}}{\partial e_{1}} \dot{e}_{1}+\frac{\partial V_{2}}{\partial e_{2}} \dot{e}_{2} \\
& =e_{1}^{T}\left[-k_{1} e_{1}+g_{11}\left(x_{1}\right) e_{2}\right]+e_{2}^{T}\left[f_{2}\left(x_{1}, x_{2}\right)+g_{2}\left(x_{1}\right) u+\rho_{2}(x)-\dot{x}_{2, d}\right] \\
& =-k_{1}\left\|e_{1}\right\|^{2}+e_{1}^{T} g_{11}\left(x_{1}\right) e_{2}+e_{2}^{T}\left[f_{2}\left(x_{1}, x_{2}\right)+g_{2}\left(x_{1}\right) u+\rho_{2}(x)-\dot{x}_{2, d}\right] \\
& =-k_{1}\left\|e_{1}\right\|^{2}+e_{2}^{T}\left[g_{11}^{T}\left(x_{1}\right) e_{1}+f_{2}\left(x_{1}, x_{2}\right)+g_{2}\left(x_{1}\right) u+\rho_{2}(x)-\dot{x}_{2, d}\right]
\end{aligned}
$$

Taking the derivative of (28) gives:

$$
\begin{aligned}
\dot{x}_{2, d} & =\frac{\partial x_{2, d}}{\partial x_{1}} \dot{x}_{1}+\frac{\partial x_{2, d}}{\partial x_{1, d}} \dot{x}_{1, d}+\frac{\partial x_{2, d}}{\partial \dot{x}_{1, d}} \ddot{x}_{1, d} \\
& =\frac{\partial x_{2, d}}{\partial x_{1}}\left[f_{1}\left(x_{1}\right)+g_{11}\left(x_{1}\right) x_{2}+\rho_{1}(x)\right]+g_{11}^{-1}\left(x_{1}\right) k_{1} \dot{x}_{1, d}+g_{11}^{-1}\left(x_{1}\right) \ddot{x}_{1, d} \\
& =\frac{\partial x_{2, d}}{\partial x_{1}}\left[f_{1}\left(x_{1}\right)+g_{11}\left(x_{1}\right) x_{2}+\rho_{1}(x)\right]+g_{11}^{-1}\left(x_{1}\right)\left[k_{1} \dot{x}_{1, d}+\ddot{x}_{1, d}\right]
\end{aligned}
$$

Inserting (34) into (33) yields:

$$
\begin{aligned}
& \dot{V}_{2}--k_{1}\left\|e_{1}\right\|^{2}+e_{2}^{T}\left\{g_{11}^{T}\left(x_{1}\right) e_{1}+f_{2}\left(x_{1}, x_{2}\right)+g_{2}\left(x_{1}\right) u+\rho_{2}(x)\right. \\
& \left.-\frac{\partial x_{2, d}}{\partial x_{1}}\left[f_{1}\left(x_{1}\right)+g_{11}\left(x_{1}\right) x_{2}+\rho_{1}(x)\right]-g_{11}^{-1}\left(x_{1}\right)\left[k_{1} \dot{x}_{1, d}+\ddot{x}_{1, d}\right]\right\}
\end{aligned}
$$

By setting $\dot{V}_{2} \leq 0$, the controller is then given by:

$$
u=g_{2}^{-1}\left(x_{1}\right)\left[-k_{2} e_{e}-g_{11}^{T}\left(x_{1}\right) e_{1}+A\right]
$$

Where,

$$
\begin{aligned}
& A=-f_{2}\left(x_{1}, x_{2}\right)-\rho_{2}(x)+\frac{\partial x_{2, d}}{\partial x_{1}}\left[f_{1}\left(x_{1}\right)+g_{11}\left(x_{1}\right) x_{2}+\rho_{1}(x)\right] \\
& +g_{11}^{-1}\left(x_{1}\right)\left[k_{1} \dot{x}_{1, d}+\ddot{x}_{1, d}\right]
\end{aligned}
$$

In which, $k_{2}$ is the diagonal gain matrix, $k_{2}=\operatorname{diag}\left\{k_{21}, k_{22}, k_{23}\right\}$, and $k_{21}, k_{22}, k_{23}>0$. Bringing (36) into (33) gives: 


$$
\dot{V}_{2}=-k_{1}\left\|e_{1}\right\|^{2}-k_{2}\left\|e_{2}\right\|^{2}<0
$$

Until now, the design of the adaptive controller with the Backstepping method is completed as follows:

$$
\left\{\begin{array}{l}
x_{2, d}-g_{11}^{-1}\left(x_{1}\right)\left[-k_{1} e_{1}-f_{1}\left(x_{1}\right)-\rho_{1}(x)+\dot{x}_{1, d}\right] \\
u=g_{2}^{-1}\left(x_{1}\right)\left[-k_{2} e_{e}-g_{11}^{T}\left(x_{1}\right) e_{1}+A\right]
\end{array}\right.
$$

Where,

$$
\begin{aligned}
& A=-f_{2}\left(x_{1}, x_{2}\right)-\rho_{2}(x)+\frac{\partial x_{2, d}}{\partial x_{1}}\left[f_{1}\left(x_{1}\right)+g_{11}\left(x_{1}\right) x_{2}+\rho_{1}(x)\right] \\
& +g_{11}^{-1}\left(x_{1}\right)\left[k_{1} \dot{x}_{1, d}+\ddot{x}_{1, d}\right]
\end{aligned}
$$

$k_{1}, k_{2}$ are both the positive definite diagonal gain matrix. According to the Lyapunov stability criterion, the control system is asymptotic stable.

Since the above design process for the adaptive controller is based on the Backstepping method, the closed-loop control system is demonstrated to be stable. Hence, the developed control law is effective to ensure the asymptotic stability of the control system.

\section{E. The design of fault-tolerant controller based on asymptotic stability of the Least Squares Support Vector Machine}

When the NSHV system develops a fault or suffers from an external disturbance, the base adaptive controller can only ensure the system stable for a while. The LSSVM is used to regress the nonlinear disturbance term. Then the identified compensation error is joined into the base controller through which the faulttolerant control of the NSHV attitude is realized. The block diagram of the fault-tolerant is shown in Fig. 3. The uncertain fault and disturbance are supplied by the fault diagnose system based on the LSSVM observer, which has been designed early.

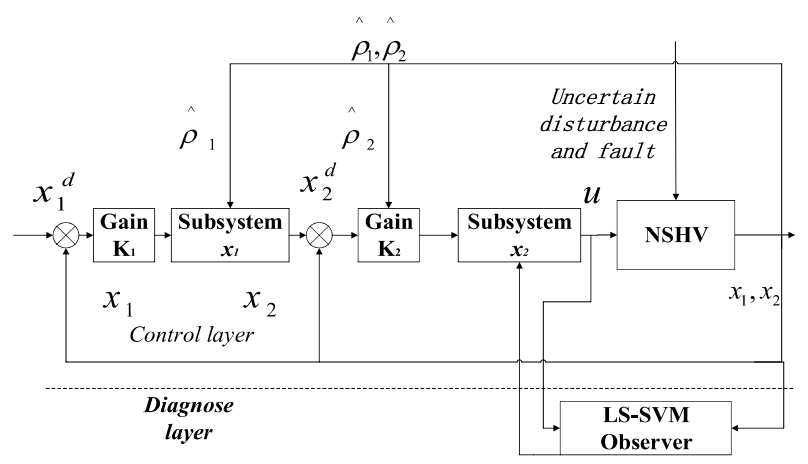

Fig. 3. Block diagram of compensator based on LSSVM. below.

The design process of the fault-tolerant is described in detail

Step 1: Design a compensator for the uncertainties based on the LSSVM for the subsystem $x_{1}$.According to the function regression principle:

$$
\hat{\rho}_{1}(x)=\sum_{i=1}^{n} \mathbf{A}_{1 i} \mathbf{K}_{1}\left(x_{i}, x_{j}\right)+\mathbf{B}_{1}
$$

Where, $\quad \mathbf{A}_{1 i}$ is a $3 \times 3$ diagonal matrix, $\mathbf{A}_{1 i}=\operatorname{diag}\left\{a_{i, 1}, a_{i, 2}, a_{i, 3}\right\}, a_{i, 1}, a_{i, 2}, a_{i, 3} \in R, \mathbf{K}_{1}\left(x_{i}, x_{j}\right)$ is a $3 \times 1$ vector formed from the Gaussian kernel function, $\mathbf{B}_{1}$ is a $3 \times 1$ vector formed from the offset quantity, and $\mathbf{B}_{1}=\left[b_{1}, b_{2}, b_{3}\right], b_{1}, b_{2}, b_{3} \in R$. The output of the subsystem $x_{1}$ is then described by:

$$
x_{2, d}=g_{11}^{-1}\left(x_{1}\right)\left[-k_{1} e_{1}-f_{1}\left(x_{1}\right)-\sum_{i=1}^{n} \mathbf{A}_{1 i} \mathbf{K}_{1}\left(x_{i}, x_{j}\right)-\mathbf{B}_{1}+\dot{x}_{1, d}\right](
$$

Step 2: Build the fault compensator based on the LSVSM for the subsystem $x_{2}$. Let us set

$$
\hat{\rho}_{2}(x)=g_{2}\left(x_{1}\right) u_{n}=g_{2}\left(x_{1}\right)\left[\sum_{i=1}^{n} A_{2 \mathbf{i}} \mathbf{K}_{2}\left(x_{i}, x_{j}\right)+\mathbf{B}_{2}\right]
$$

Where, $\quad \mathbf{A}_{2 i}$ is a $3 \times 3$ diagonal matrix, $\mathbf{A}_{2 i}=\operatorname{diag}\left\{a_{i, 1}, a_{i, 2}, a_{i, 3}\right\}, \quad a_{i, 1}, a_{i, 2}, a_{i, 3} \in R, \mathbf{K}_{2}\left(x_{i}, x_{j}\right)$ is a $3 \times 1$ vector formed from the Gaussian kernel function, $\mathbf{B}_{2}$ is the $3 \times 1$ vector formed from the Gaussian kernel function, $\mathbf{B}_{2}=\left[b_{1}, b_{2}, b_{3}\right], b_{1}, b_{2}, b_{3} \in R$. Now the output of the subsystem $x_{2}$ is described as follows:

$$
\left\{\begin{aligned}
u= & g_{2}^{-1}\left(x_{1}\right)\left[-k_{2} e_{e}-g_{11}^{T}\left(x_{1}\right) e_{1}+A^{*}\right] \\
A= & -f_{2}\left(x_{1}, x_{2}\right)-\rho_{2}(x)+\frac{\partial x_{2, d}}{\partial x_{1}}\left\{f_{1}\left(x_{1}\right)+\right. \\
& \left.g_{11}\left(x_{1}\right) x_{2}+g_{2}\left(x_{1}\right)\left[\sum_{i=1}^{n} A_{2 \mathbf{i}} \mathbf{K}_{2}\left(x_{i}, x_{j}\right)+\mathbf{B}_{2}\right]\right\} \\
& +g_{11}^{-1}\left(x_{1}\right)\left[k_{1} \dot{x}_{1, d}+\ddot{x}_{1, d}\right]
\end{aligned}\right.
$$

In summary, when the NSHV system suffers from uncertain faults or disturbance, the control system identifies them through the LSSVM strategy and sends the outcomes to the subsystem $x_{1}$ and $x_{2}$. So far, the design procedures of the controller based on the Backstepping method have been completed to ensure the NSHV system stable.

\section{SimULATION RESULTS}

In the simulations, the LSSVM uses the RBF as the kernel function. The inputs of training samples are the state variables $x_{1}, x_{2}$, and the output is the system fault. To verify the validity and reliability of the observer, that the closed-loop system of the attitude control breaks down at 10 second to simulate the two kinds of fault: 1) Mutational system fault, i.e., $u(x, v, t)$ is 
$[3,0,0,0,0,0], 2)$ Slow system fault given in the form of $\left[\sin x_{1}+x_{2}, 0,0,0,0,0\right]$. The observer gain matrix $G$ is set as:

$$
G=\left[\begin{array}{lllllc}
-9.6171 & 1.0000 & -1.2244 & 0 & 0 & 0 \\
-12.3887 & -2.1147 & -0.0690 & 0 & 0 & 0 \\
0.5212 & 0 & -10.8539 & 1.0000 & 0 & 0 \\
-5.4672 & 0 & -20.3576 & -1.4142 & 0 & 0 \\
0 & 1.0000 & 0 & 0 & -0.5858 & 1.0000 \\
0 & 0 & 0 & 0 & -0.4216 & -1.4142
\end{array}\right]
$$

The estimated value and the true value of the system fault in case 2 and 2 are given in Fig. 4 and Fig. 5, respectively.

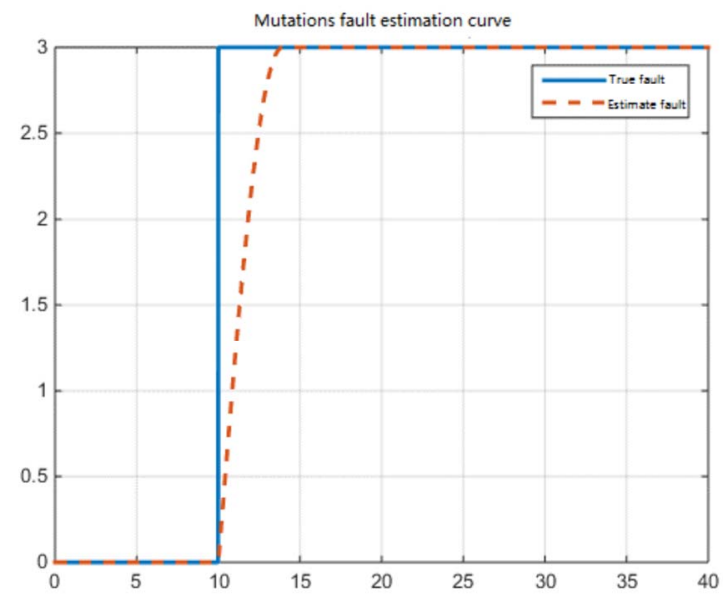

Fig. 4. Estimation curve (The red dotted line) and true curve (The blue line) of mutational fault case.

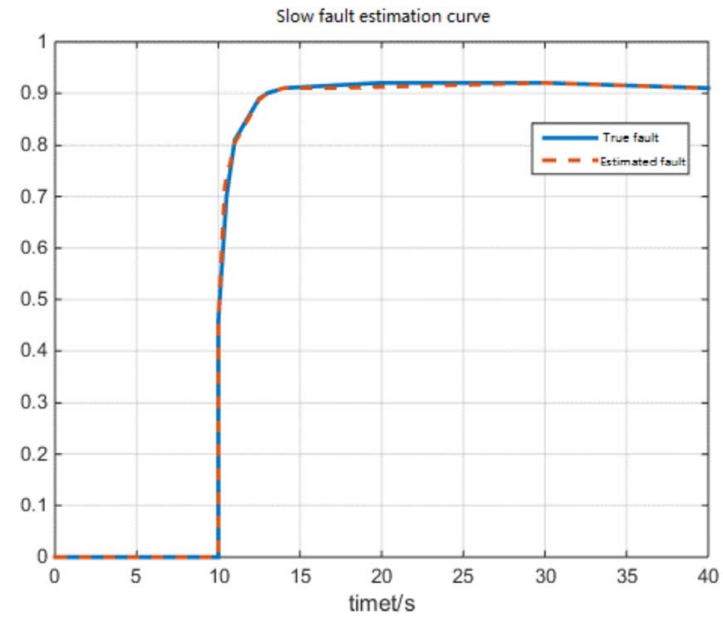

Fig. 5. Estimation curve (The red dotted line) and true curve (The blue line) of slow fault case.

Fig. 4 and Fig. 5 demonstrates that, when a mutational fault and slow fault occur, the system can estimate the fault mode and track the true failure quickly through the LSSVM, which tends to the actual system failure at the end. So the observer based on the LSSVM can track system failure quickly and efficiently to meet the requirements of the system fault diagnosis.

The validity of the reentry attitude fault-tolerant controller with the model of the fault-tolerant control system is performed in Matlab environment. In the simulation, the initial conditions are set as: height $H_{0}=65 \mathrm{~km}$, velocity $V_{0}=6100 \mathrm{~km} / \mathrm{s}$, the initial attack angle, the sideslip angle, and the dead reckoning angle are all 0.0 , i.e., $\alpha_{0}=\beta_{0}=\mu_{0}=0$. The initial velocity of the roll, pitch, and yaw angle are all 0.0,i.e., $p_{0}=q_{0}=r_{0}=0$.

For the subsystem $\rho_{1}(x), \quad C=1345.5, P=1.9252$, and for the subsystem $\rho_{2}(x), C=1533.6, P=2.2163$. For the base controller obtained via the Backstepping methods, $k_{1}=\operatorname{diag}\{4,6,5\}$, $k_{2}=\operatorname{diag}\{6,8,9\}$.

In order to verity the reliability and effectiveness, the fault information is added and the simulation verification for the two flight states of the NSHV is made as below.

1) Set the tracking instruction of the attitude angle as $x_{1, d}=\left[\alpha^{d}, \beta^{d}, \mu^{d}\right]^{T}=\left[4^{\circ}, 0^{\circ}, 3^{\circ}\right]$. In the loop of the angular rate, the deviation of elevator, aileron and rudder are taken as $0.5^{\circ}, 0.1^{\circ}, 0.1^{\circ}$. The control matrix is manipulated to affect the moment matrix first, and then affect the flight state of the NSHV.

The NSHV system breaks down at $t=10 \mathrm{~s}$. The, response curve of the reentry attitude angle is shown in Fig.6.

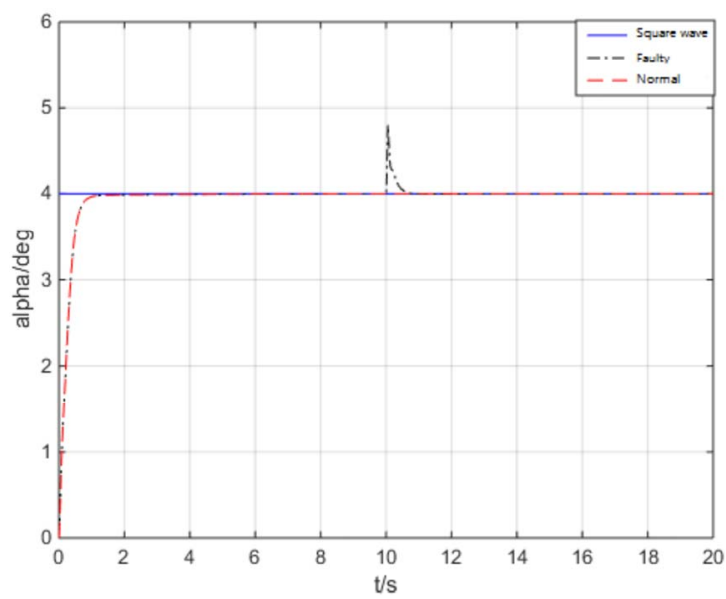

Fig. 6. Response curve of the attack angle. 


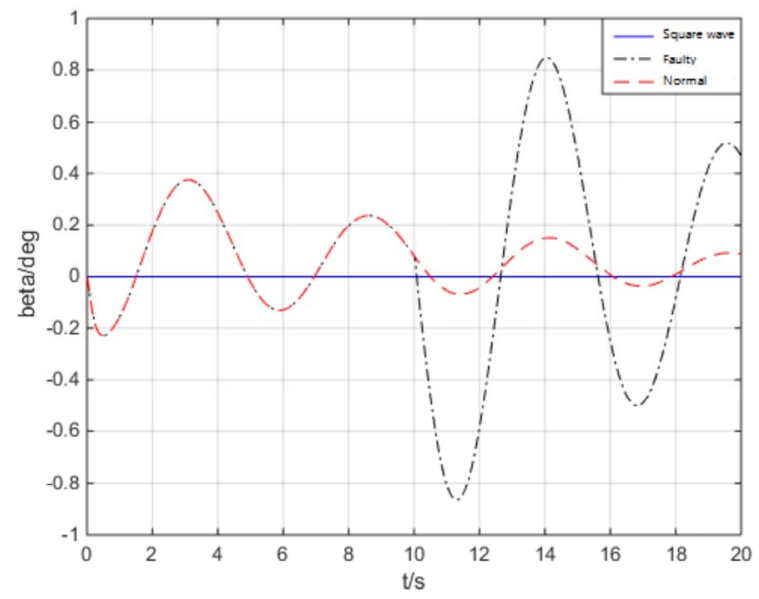

Fig. 7. Response curve of the sideslip angle.

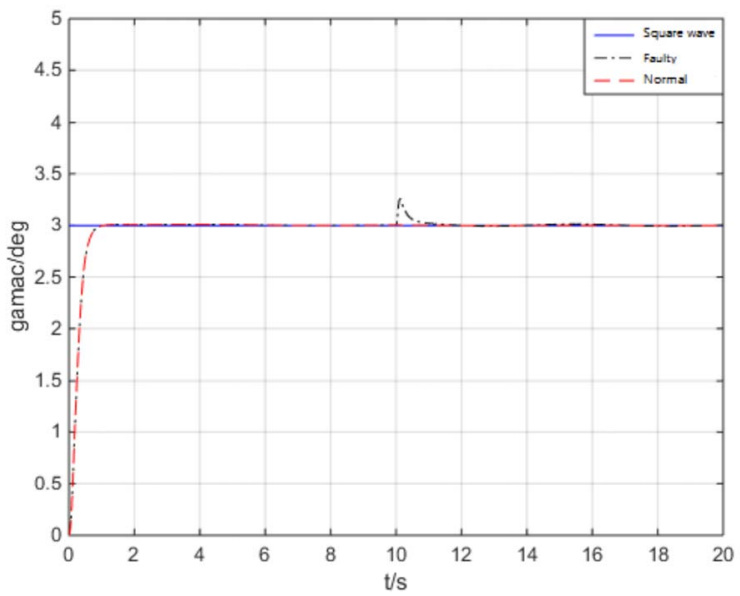

Fig. 8. Response curve of the dead reckoning angle.

2) Set the square wave as the tracking instruction for attitude angle control of the NSHV, in the loop of attitude angle control. The outer interference is simulated by perturbing the sideslip angle to the degree of $10 \%, \rho_{1}(x)=[0,0.1 \beta, 0]^{T}$, The fault is set by $u_{n}=\left\{\begin{array}{l}0, t<10 \\ {[0.3,0.1,0.05], t \geq 10}\end{array}\right.$, then $\hat{\rho}_{2}(x)=g_{2}\left(x_{1}\right) u_{n}$.

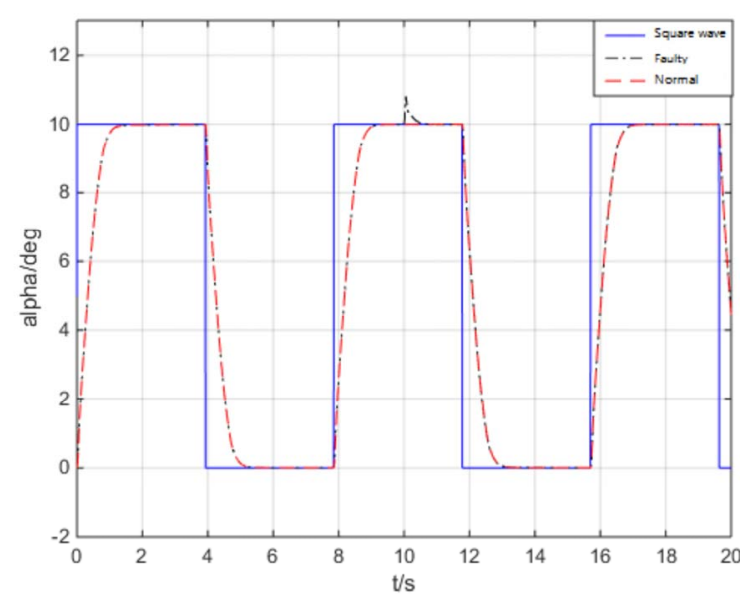

Fig. 9. Response curve of the attack angle.

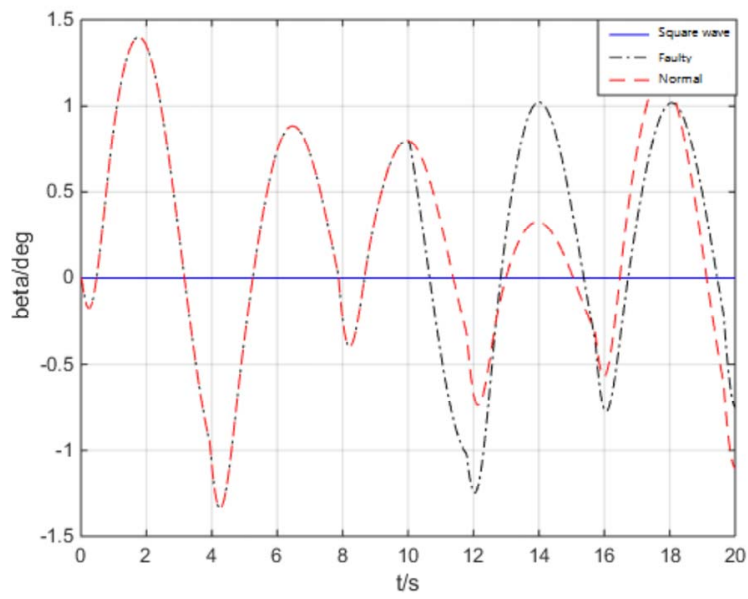

Fig. 10. Response curve of the sideslip angle.

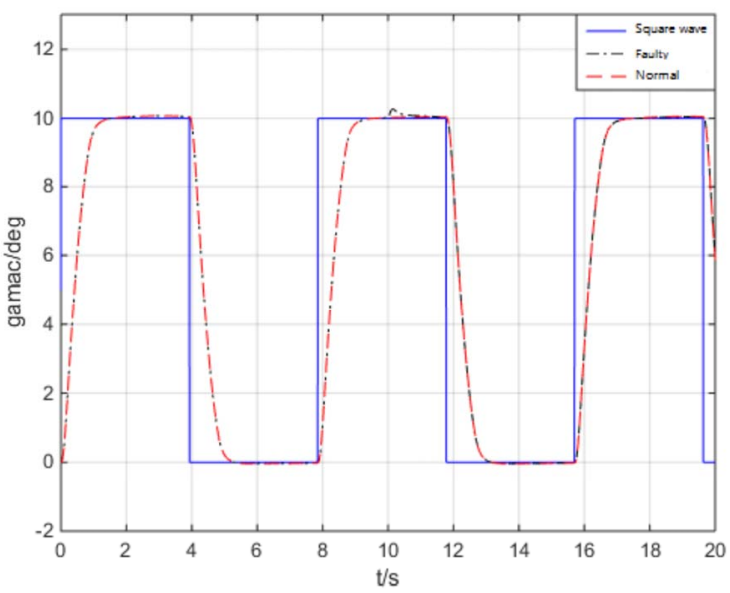

Fig. 11. Response curve of the dead reckoning angle. 
When a fault occurs at $\mathrm{t}=10 \mathrm{~s}$, the response curves of the reentry attitude angles are given in Figs.9-11.

Where the blue line is the instruction of the attitude angle, the red line is the response curve in the normal situation, and the black line is the response curve in the faulty situation. The results show that, when it is in the normal situation, the system is stable, the attitude angle can respond to the input and output in time. When a fault occurs, the attitude angles fluctuate at different levels. If not taking right control actions, the flight system will fall unstable. The figures show that the attitude angle tends to stabilize quickly after the fluctuation, that is because it takes some time to train the samples using the LSSVN method. At the moment, the output of the compensator is 0 , the flight control system can just keep stable for a while with a small fluctuation due to the base controller. After the LSSVM system finishes the process of identifying the fault information in the NSHV, the compensator adds the fault information to the Backstepping base controller. After that the base controller adjusted itself to keep the control system stable again and achieve the purpose of the fault-tolerant control.

In summary, the adaptive fault-tolerant controller with the compensation through the LSSVM technique is effective in dealing with the faults happening in the process of the reentry stage of the NSHV. When the NSHV system breaks down, the control system has a certain robustness, to detect the fault online adjust the attitude of the NSHV with the adaptive controller, for improving the dynamic property of the vehicle in fault situation.

\section{CONCLUSION}

In this paper, the first an attitude observer has been designed for the reentry process of the NSHV by using the LSSVM technique which uses the principle of least squares support vector machine (SVM) regression function. Then an adaptive faulttolerant controller has been designed through the Backstepping method,. The controller approaches the fault information and interference information online, and then adds the identified fault model to the adaptive base controller for achieving the aim of fault-tolerant control. Finally we simulated with Matlab and demonstrated that when fault occurred, the flight attitude could still follow the command instructions well. This has illustrated the proposed fault-tolerant control strategy designed through the method of the LSSVM and the Backstepping, is effective. The control strategy has met the aim of fault-tolerant control at a sudden failure, and kept the vehicle stable. Furthermore, the fault-tolerant control strategy we have presented in this paper can not only benefit for the NSHV, but also for the general aviation, marine and other industrial applications[19].

\section{ACKNOWLEDGMENT}

Special thanks are made for the support of the National Science Foundation project (Grant No. 51206007), Aeronautical Science Foundation of China (Grant No. 2013ZC51), China Scholarship Council (CSC) (Grant No.20140625104), and to all the colleagues who have made constructive suggestions and offered the research supports throughout this work.

\section{REFERENCES}

[1] Jorris T R. Common aero vehicle autonomous reentry trajectory optimization satisfying waypoint and no-fly zone constraints [D]. Alabama: Air University 2007

[2] Vijay G, Ben Ali Bdira E, Ibnkahla M. Congnition in wireless sensor networks: A perspective [J]. IEEE Sensors Journal, 2011, 11(3): 582-592

[3] Qu li, Liu Guohai, Zhang Hao, et al. Decoupling Control for Five-Phase FaultTolerant Permanent-Magnet Motor By using SVM Inverse System Method [C]. 2014 International Joint Conference on Neural Networks, Beijing, China, 2014

[4] Sun Qiaomei, Ren Guang, Yue jin, et al. SVM Inverse Model-based Heading Control of Unmanned Surface Vehicle [C]. 2010 IEEE Youth Conference on Information Computing and Telecommunications, Beijing, China, 2010

[5] Shaughnessy J. D., Pinckney S. Z. and McMinn J. D. Hypersonic Vehicle Simulation Model: Winged-Cone Configuration[J].NASA Langley Research Center, 1990.

[6] Shahriar K. and Richard C. Development of An Aerodynamic Database for A Generic Hypersonic Air Vehicle[C]. AIAA Guidance, Navigation, and Control Conference and Exhibit, 2005, pp. 2005-6257.

[7] Tong, Shaocheng, Baoyu Huo, and Yongming Li. Observer-based adaptive decentralized fuzzy fault-tolerant control of nonlinear large-scale systems with actuator failures[J].Fuzzy Systems, IEEE Transactions on 22.1 (2014): $1-15$.

[8] Shen, Qikun, Bin Jiang, and Vincent Cocquempot. Fuzzy logic system-based adaptive fault-tolerant control for near-space vehicle attitude dynamics with actuator faults[J]. Fuzzy Systems, IEEE Transactions on 21.2 (2013): 289-300.

[9] Bustan, Danyal, S. K. Sani, and Naser Pariz. Adaptive fault-tolerant spacecraft attitude control design with transient response control[J].Mechatronics, IEEE/ASME Transactions on 19.4 (2014): 1404-1411.

[10]Psarakis, Mihalis, and Andreas Apostolakis. Fault tolerant FPGA processor based on runtime reconfigurable modules[C].Test Symposium (ETS), 2012 17th IEEE European. IEEE, 2012.

[11]Stilwell D J. State-space interpolation for a gain-scheduled autopilot [J] Journal of Guidance, Control, and Dynamics, 2001, 24(3): 460-465.

[12]Zhao Jing, Jiang Bin, Shi Peng, et al. Fault-tolerant Control Design Based on Adaptive Sliding Mode Technique for Near Space Vehicles [C]. 2012 24th Chinese Control and Decision Conference, Taiyuan, China, 2012: 1618-1623

[13]Ye D, Yang G H. Adaptive fault-tolerant tracking control against actuator faults with application to flight control [J]. IEEE Transactions on Control Systems Technology, 2006, 14 (6): 1088-1096

[14]Zhang X D, Parisini T, Marios M P. Adaptive fault-tolerant control of nonlinear uncertain systems: an information-based diagnostic approach [J]. IEEE Trans. Automatic Control, 2004, 49(8):1259-1274

[15]Tao G, Joshi S M, Ma X L. Adaptive state feedback and tracking control of systems with actuator failures [J]. IEEE Transactions on Automatic Control, 2001, 46(1): 78-95

[16]Zhao Jing, Jiang Bin, Zhen He, et al. Adaptive Backstepping Fault Tolerant Control for Near Space Vehicles [C]. Proceedings of the 33rd Chinese Control Conference, Nanjing, China, 2014: 3053-3058

[17]Jiang Y, Hu Q, Ma G. Adaptive backstepping fault-tolerant control for flexible spacecraft with unknown bounded disturbances and actuator failures [J]. ISA Transactions, 2009, pp. 1-13.

[18]Song Jia, Qi Xiaoqiang. Supercavitating Vehicle LSSVM Multiple Intelligent Control. Intelligent Information Technology Application Association.Proceedings of the 2nd International Conference on Applied Mechanics and Mechanical Engineering(ICAMME 2011 Part1)[C].Intelligent Information Technology Application Association:,2011:6.

[19] Yang, Erfu, and D.Gu. Nonlinear Formation-Keeping and Mooring Control of Multiple Autonomous Underwater Vehicles [J]. IEEE/ASME Transactions on Mechatronics 12.2(2007):164-178. 